Chelsey L. Dunham

Department of Biomedical Engineering,

Washington University in St. Louis,

St. Louis, M0 63130

e-mail: chelsey.dunham@wustl.edu

Ryan M. Castile

Department of Mechanical Engineering and

Materials Science,

Washington University in St. Louis,

St. Louis, M0 63130

e-mail: castiler@wustl.edu

\section{Aaron M. Chamberlain}

Department of Orthopaedic Surgery,

Washington University in St. Louis,

St. Louis, M0 63130

e-mail: amchamberlain@wustl.edu

Leesa M. Galatz

Department of Orthopaedic Surgery, Mount Sinai Hospital,

New York, NY 10029

e-mail: leesa.galatz@mountsinai.org

\section{Spencer P. Lake}

Mem. ASME

Department of Mechanical Engineering and Materials Science,

Washington University in St. Louis,

St. Louis, M0 63130;

Department of Orthopaedic Surgery, Washington University in St. Louis,

St. Louis, M0 63130;

Department of Biomedical Engineering,

Washington University in St. Louis,

1 Brookings Drive,

Campus Box 1185

St. Louis, M0 63130

e-mail: lake.s@wustl.edu

\section{Pronation-Supination Motion Is Altered in a Rat Model of Post- Traumatic Elbow Contracture}

The elbow joint is highly susceptible to joint contracture, and treating elbow contracture is a challenging clinical problem. Previously, we established an animal model to study elbow contracture that exhibited features similar to the human condition including persistent decreased range of motion (ROM) in flexion-extension and increased capsule thickness/adhesions. The objective of this study was to mechanically quantify pronation-supination in different injury models to determine if significant differences compared to control or contralateral persist long-term in our animal elbow contracture model. After surgically inducing soft tissue damage in the elbow, Injury I (anterior capsulotomy) and Injury II (anterior capsulotomy with lateral collateral ligament transection), limbs were immobilized for 6 weeks (immobilization (IM)). Animals were evaluated after the IM period or following an additional 6 weeks of free mobilization (FM). Total ROM for pronation-supination was significantly decreased compared to the uninjured contralateral limb for both IM and FM, although not different from control limbs. Specifically, for both IM and FM, total ROM for Injury I and Injury II was significantly decreased by $\sim 20 \%$ compared to contralateral. Correlations of measurements from flexion-extension and pronation-supination divulged that FM did not affect these motions in the same way, demonstrating that joint motions need to be studied/treated separately. Overall, injured limbs exhibited persistent motion loss in pronation-supination when comparing side-toside differences, similar to human post-traumatic joint contracture. Future work will use this animal model to study how elbow periarticular soft tissues contribute to contracture. [DOI: $10.1115 / 1.4036472]$

\section{Introduction}

Traumatic injury to the elbow (e.g., dislocation and fracture) often leads to subsequent post-traumatic joint contracture. More so than other joints, the elbow is especially susceptible to contracture making it a common and challenging clinical problem. The elbow is anatomically and biomechanically one of the most complex joints in the body [1,2] due to the highly congruent joint surfaces of the three bones that create the joint [3]. Articulation of these bones is stabilized by periarticular soft tissues (i.e., capsule, ligaments, tendons, and muscles), which are often disrupted when the elbow is injured. Damage to these tissues is poorly tolerated and even a relatively minor injury can result in significant functional impairment, making it difficult to predict who is at risk for developing contracture [4]. Due to the clinical challenge of managing elbow injuries, there is a need to develop an animal model of post-traumatic elbow contracture to understand its etiology and to ultimately develop new intervention and treatment strategies, which is not possible with a clinical study.

Previously, our group developed an animal model of elbow post-traumatic joint contracture in Long-Evans rats by surgically

Manuscript received November 30, 2016; final manuscript received April 7 , 2017; published online June 6, 2017. Assoc. Editor: Eric A Kennedy. creating clinically relevant soft tissue injuries followed with 6 weeks of unilateral joint immobilization [5,6]. Our animal model exhibited joint motion loss in flexion-extension and changes to the joint capsule which were consistent with patterns reported for human tissue [5]. At the end of the 6 weeks of immobilization, the constraint was removed to allow FM of the joint for an additional 6 weeks. Importantly, joint motion loss in flexion-extension and capsular changes persisted even after the FM period, providing further validation for this animal model of joint contracture [6]. Our previous work only evaluated the limb in flexion-extension; however, the elbow plays a critical role in enabling pronation-supination (P-S) motion of the forelimb (Fig. 1).

A majority of current work on post-traumatic joint contracture focuses only on dysfunction in flexion-extension [7-9]. However, both flexion-extension and $\mathrm{P}-\mathrm{S}$ are clinically significant motions, essential for most daily and vocational activities. For P-S, the range of motion necessary for daily living in humans is $\sim 50 \mathrm{deg}$ of motion in both supination and pronation [9]. The shoulder can compensate to a limited extent for pronation loss but no upper extremity movement can replace supination [10]. Motion loss in $\mathrm{P}-\mathrm{S}$ is not consistently or completely restored using common therapies applied to restore motion loss in flexion-extension, and thus, recovery of $\mathrm{P}-\mathrm{S}$ may require a more invasive and higher-risk surgical intervention [11]. The ability to identify improvements 


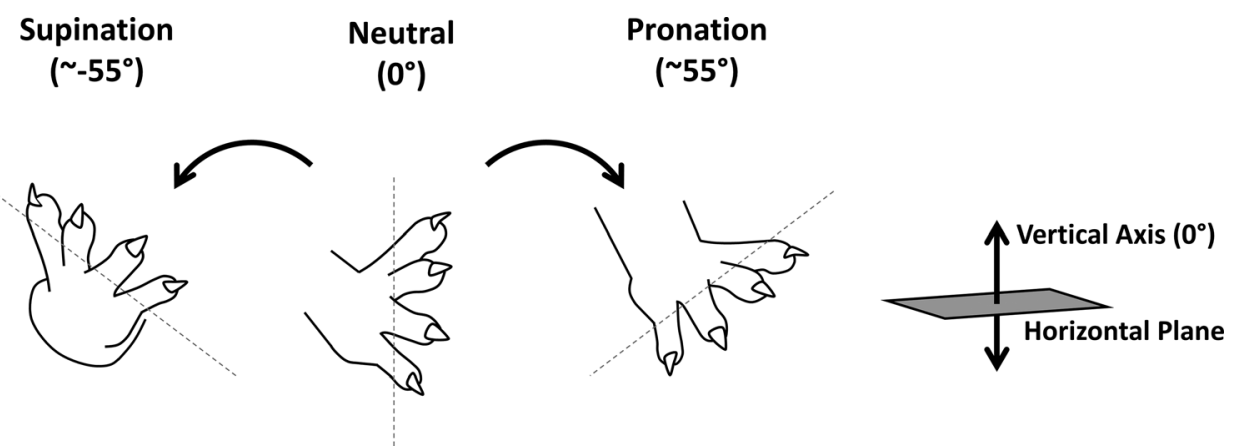

Fig. 1 Schematic of pronation-supination motion in the rat forelimb

for therapies in $\mathrm{P}-\mathrm{S}$ was previously limited by the lack of an appropriate animal model.

Studying P-S in previous animal models was not possible due to the structure and function of the joint studied (i.e., knee) $[10,12,13]$. However, our animal model is specific to the elbow and, unlike other models, has the ability to evaluate P-S [5]. Furthermore, a key criterion that informed the selection of Long-Evans rats for our animal model was the degree to which the forelimb of this breed of rat can pronate and supinate, which is similar to patterns in humans for given sets of functional tasks (i.e., forward reach and return food to mouth) [14-16]. Our previous study only evaluated flexion-extension; therefore, the objective of this study was to mechanically quantify $\mathrm{P}-\mathrm{S}$ in different injury models to determine if significant differences compared to control or contralateral persist long-term in our animal elbow contracture model. We hypothesized that P-S motion would be altered similarly as our results in flexion-extension, specifically that the most severe injury model would experience the largest amount of motion loss compared to control and contralateral, which would persist even following free mobilization of the joint.

\section{Methods and Materials}

Animal Model. Long-Evans rats (Charles River Laboratories International, Wilmington, MA) were used based on criteria previously described [5,6]. Briefly, these animals were selected because of similarities to human anatomy, functional ROM, and use of their upper extremities. Functionally, Long-Evans rats not only exhibit flexion-extension but also P-S, which differentiates them from other animal species we considered and even other breeds of rats [14-17]. Specifically, Long-Evans rats supinate their paws during feeding, pronate during reaching, and show overall forelimb rotation throughout other cage activities [14,16]. Since both flexion-extension and $\mathrm{P}-\mathrm{S}$ are clinically significant motions of the human elbow, similarities of joint motions to humans were an important factor in selecting Long-Evans rats for the animal model utilized in this study.

Injury Model. This study was approved by our university's Institutional Appropriate Care and Animal Use Committee (IACUC). The experimental design was similar to what was previously described [5,6], in which male Long-Evans rats (250-300 g, 8-10 weeks old) were randomized into three surgical groups (Sham, Injury I, and Injury II) and a group of age-matched control animals. Animals in each group were anesthetized and surgery was performed on the left limb by an orthopedic surgeon: Sham (superficial lateral incision without violation of joint structures), Injury I (anterior capsulotomy), and Injury II (anterior capsulotomy with lateral collateral ligament transection) (Fig. 2). Sham animals were evaluated to understand the effect of joint immobilization without periarticular joint tissue injury. Animals received single doses of antibiotic $(7.5 \mathrm{mg} / \mathrm{kg}$ enrofloxacin; Bayer Health LLC, Shawnee Mission, KS) and nonsteroidal anti-inflammatory drug (NSAID) $(5 \mathrm{mg} / \mathrm{kg}$ carprofen; Pfizer Animal Health, New York, NY) preoperatively via subcutaneous injection and one dose of analgesic $(0.5 \mathrm{cc}$ of $5 \mathrm{mg} / \mathrm{mL}$ bupivacaine; Hospira, Lake Forest, IL) postoperatively via subcutaneous injection under the closed incision. Contralateral (CL) and control limbs served as comparisons to injured/immobilized limbs.

After surgery, injured limbs were immobilized in flexion for 6 weeks using a protocol described previously [5,6], while CL limbs and control animals were allowed unrestricted motion (Fig. 2). Animals were checked $5 \times /$ week to ensure the injured limb was constrained and to identify any signs of pain or distress. When an animal's injured limb was rewrapped, any sores or cuts caused by scratching or rubbing of the bandaging restraints were treated topically with antibiotic powder/cream (nitrofurazone (Neogen Corporation, Lexington, KY); silver sulfadiazine (Dr. Reddy's

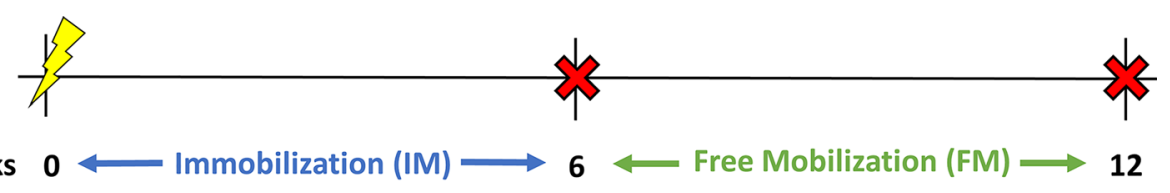

\begin{tabular}{|l|l|}
\hline \multicolumn{1}{|c|}{ Group } & \multicolumn{1}{|c|}{ Injury } \\
\hline Control & No injury or IM, normal cage activity \\
\hline Sham & Superficial lateral incision without violation of joint structures with IM \\
\hline Injury I & Anterior capsulotomy with IM \\
\hline Injury II & Anterior capsulotomy \& LCL transection with IM \\
\hline
\end{tabular}

Fig. 2 Schematic of the experimental method timeline and a table defining each experimental animal-injury group (lightning bolt $=$ surgery and $X=$ analysis time point) 


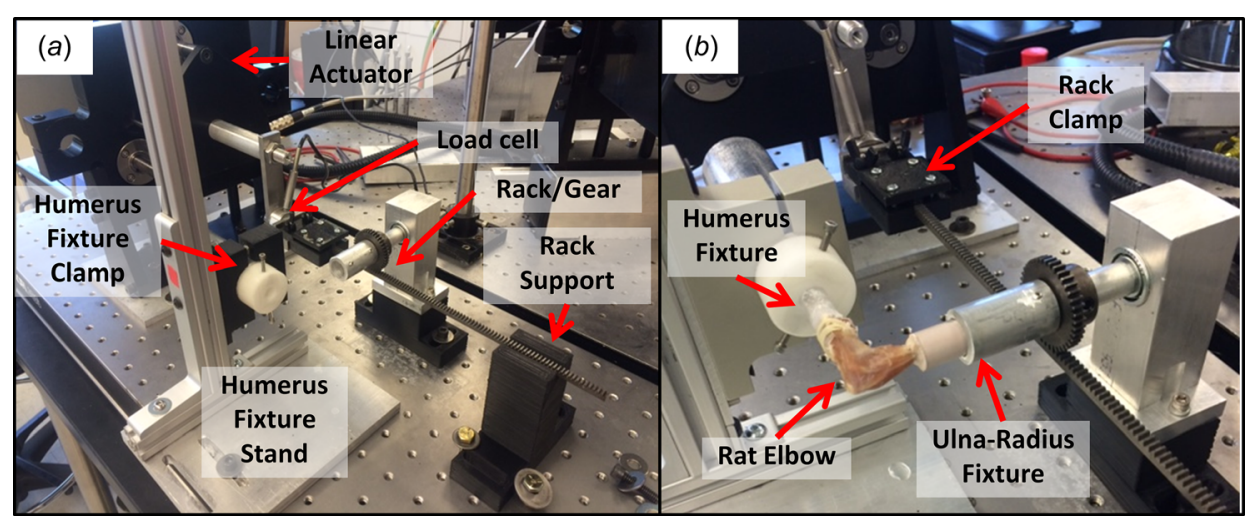

Fig. 3 (a) The biomechanical test system uses a rack and pinion gear to convert linear displacement to rotational cyclic loading of the rat elbow in pronation-supination. Specimens were secured with ulna-radius and humerus fixtures. A load cell was used to measure the axial force. (b) The test system loaded with a rat elbow.

Laboratories Louisiana, Shreveport, LA)) and/or chaffing cream (Prestige Brands, Tarrytown, NY). After 6 weeks of immobilization, half of the animals were sacrificed (IM) and half had their bandages removed and were allowed unrestricted cage activity for an additional 6 weeks (FM) (Fig. 2). After either the immobilization or free mobilization period, animals were sacrificed via $\mathrm{CO}_{2}$ inhalation and immediately stored in a $-20^{\circ} \mathrm{C}$ freezer.

Across the four groups (Injury I, Injury II, Sham, and control), this study used a total of 60 male Long-Evans rats with $n=6$ / group for IM and $n=9 /$ group for FM. Three and four animals were excluded from the IM and FM time points, respectively, due to dissection/sample preparation (i.e., limb potting issues) and testing abnormalities (i.e., outliers due to extreme rotation and nonsmooth rotation of the limb outside of the system prior to mechanical testing). After removing these few samples, a total of 93 limbs were included in the dataset.

Mechanical Testing. Mechanical testing was performed on both injured and CL limbs from each animal as well as unilateral limbs from controls. Forelimbs were prepared for mechanical testing as described previously [5,6]. Limbs were first loaded in flexion-extension and then in P-S (flexion-extension mechanical data were published previously $[5,6])$. A preliminary study that repeated flexion-extension testing after P-S testing ( $n=20$ animals; age- and sex-matched) confirmed that no joint damage occurred in the initial mechanical testing that would affect the joint mechanics in P-S. Consistent flexion-extension results before and after $\mathrm{P}-\mathrm{S}$ testing also demonstrated that no joint damaged occurred as a result of $\mathrm{P}-\mathrm{S}$ mechanical testing.

A novel mechanical test setup was designed and built to evaluate rat elbow stiffness and joint contracture in P-S (Fig. 3(a)). To the best of our knowledge, it is the first system to quantify P-S joint motion in an animal model. The P-S test setup is a modification of the equipment utilized in our flexion-extension test system [5]. A linear actuator and load cell are used to apply a linear displacement and measure the force, respectively (TestResources, Shakopee, $\mathrm{MN})$. A vertical rack and pinion gear converts the linear displacement to rotational motion, which allows for load-controlled cyclic testing. In the test setup, the limb was potted so the radius and ulna could move relative to each other, which allowed smooth articulation of the joint (Fig. 3(b)). At the beginning of the test, the joint was placed in $\sim 90$ deg flexion with the paw in a neutral position of $\sim 0$ deg (Fig. 1). After the limb was secured, it was cyclically loaded to $\pm 0.85 \mathrm{~N}$ (corresponding to $\pm 11.75 \mathrm{Nmm}$ of torque) for five cycles at $0.3 \mathrm{~mm} / \mathrm{s}$. Preliminary testing was used (1) to determine how to align the limb in a neutral ( $0 \mathrm{deg})$ starting position, (2) to ensure the limb rotational axis in the test setup was centered on the applied torque, and (3) to determine that the torque limits were sufficient to mechanically probe $\mathrm{P}-\mathrm{S}$ motion, without damaging tissues of the elbow joint.

Force and displacement data from the fifth cycle were converted to torque and angular position for $\mathrm{P}-\mathrm{S}$ data. Torque-angle curves were analyzed with a custom written MATLAB program (Mathworks, Natick, MA) to quantify elbow joint motion in P-S (Fig. 4(a)). P-S measurements included total ROM, neutral zone length, neutral zone stiffness, pronation stiffness, and supination stiffness; similar parameters were previously defined for loading curves of flexion-extension tests [5,6]. Briefly, the neutral zone is the flatter/linear region of the curve that falls between the loading and unloading curves of pronation and supination. The neutral zone is a clinically relevant parameter because it represents the functional range of motion, specifically it is the amount of motion possible before a larger force is required for further joint movement $[5,6]$. Stiffness is defined as the linear region of the loading curves for pronation and supination and the average of the loading and unloading curves for the neutral zone [5,6]. It is calculated as the slope of a linear fit of these regions. Average loading curves were also created by averaging the points of maximum supination, maximum pronation, and both end points of the neutral zone stiffness/ length curve within each group to show qualitative representations of joint motion. Group-averaged values were used to plot average curves for each group (control, Sham, Injury I, and Injury II) for both IM and FM.

Statistical Analysis. One-way analysis of variance (ANOVA) tests compared mechanical test parameters between (1) control and injured limbs for each group, and (2) control and CL limbs for each group, enabling comparisons of injured-only and CL-only groups. ANOVAs were also separated because injured and CL limbs are not independent measures. When significance was found during the ANOVA analyses $(p<0.05)$, post-hoc $t$-tests with Bonferroni corrections were used to compare each experimental group to control. Side-to-side limb differences were evaluated with paired $t$-tests to compare injured limbs to CL limbs within each group. Correlations between mechanical testing parameters in flexion-extension $[5,6]$ and $\mathrm{P}-\mathrm{S}$ were completed with data from all groups (i.e., Sham, Injury I, Injury II, control, and CL limbs) in order to identify relationships between different types of joint motion in our animal model. All statistical analyses were performed in GRAPHPAD PRISM (GraphPad Software Inc., La Jolla, CA).

\section{Results}

Qualitatively, the group-averaged mechanical test data exhibited differences in the overall loading profiles when comparing each injured group to its respective uninjured CL group (Figs. 4(b) and 4(c)). Average curves for the injured limbs (i.e., dashed lines) 

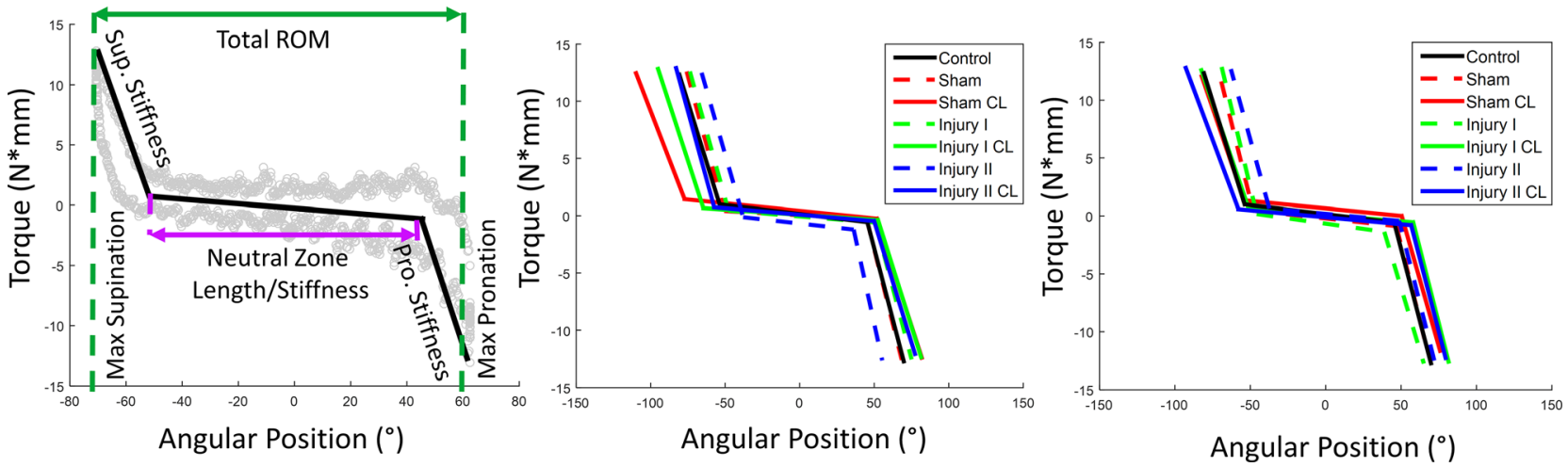

Fig. 4 (a) Schematic of the torque-angle loading curve with parameters quantified for pronation-supination. The light gray circles are representative data. The black line for neutral zone stiffness/length is the average of the loading and unloading curves. Average curves for each injury group and contralateral for pronation-supination in $(b)$ IM and $(c)$ FM. These average curves represent the mean of all the animals tested per group without the standard deviation to make the differences between curves more apparent (dashed lines = injured limb; solid line = contralateral; solid black line = control).

Table 1 Quantitative results for pronation-supination joint mechanics: total range of motion (ROM) and neutral zone length values for immobilization (IM) and free mobilization (FM)

\begin{tabular}{|c|c|c|c|c|c|}
\hline & & \multicolumn{2}{|c|}{ Total ROM (deg) } & \multicolumn{2}{|c|}{ Neutral zone length (deg) } \\
\hline & & Immobilization & Free mobilization & Immobilization & Free mobilization \\
\hline Control & $n=4 \mathrm{IM}, 9 \mathrm{FM}$ & $159.7 \pm 17.7$ & $143.8 \pm 26.3$ & $107.1 \pm 21.0$ & $91.3 \pm 13.4$ \\
\hline Sham & $n=4 \mathrm{IM}, 6 \mathrm{FM}$ & $145.3 \pm 10.6$ & $137.6 \pm 34.2$ & $95.6 \pm 11.1$ & $95.1 \pm 25.4$ \\
\hline Sham CL & $n=5 \mathrm{IM}, 7 \mathrm{FM}$ & $193.0 \pm 35.6$ & $158.9 \pm 27.3$ & $129.1 \pm 23.1$ & $104.5 \pm 24.1$ \\
\hline Injury I & $n=6 \mathrm{IM}, 8 \mathrm{FM}$ & $149.1 \pm 19.9$ & $134.6 \pm 26.6$ & $98.8 \pm 20.0$ & $84.7 \pm 20.8$ \\
\hline Injury I CL & $n=6 \mathrm{IM}, 8 \mathrm{FM}$ & $177.4 \pm 25.3$ & $164.6 \pm 20.3$ & $117.2 \pm 19.1$ & $109.1 \pm 20.8$ \\
\hline Injury II & $n=6 \mathrm{IM}, 8 \mathrm{FM}$ & $122.7 \pm 46.6$ & $136.0 \pm 13.9$ & $74.2 \pm 34.8$ & $86.2 \pm 13.4$ \\
\hline Injury II CL & $n=6 \mathrm{IM}, 8 \mathrm{FM}$ & $161.1 \pm 52.2$ & $173.0 \pm 25.5$ & $107.3 \pm 41.0$ & $114.6 \pm 21.6$ \\
\hline
\end{tabular}

Note: Average \pm standard deviation; bolded values $=$ significantly different from contralateral limb; $p<0.05$.

No values were significantly different from control.

showed less total ROM and shorter neutral zone length compared to CL curves (i.e., solid lines) following IM, indicating side-to-side differences in $\mathrm{P}-\mathrm{S}$ joint motion in this model. Side-to-side differences persisted following FM, illustrating that $\mathrm{P}-\mathrm{S}$ motion remains altered even after a period of joint free mobilization. Injury II exhibited the most severe differences compared to CL for both IM and FM. Interestingly, CL curves exhibited increased total ROM compared to the control curve at both IM and FM.

Quantitatively, P-S mechanical testing identified significant alterations in side-to-side differences but did not exhibit significant changes compared to control in our animal model of post-traumatic joint contracture. After immobilization, Injury II total ROM was significantly smaller than its CL limb $(p=0.030)$, while Injury I and Injury II neutral zone length were significantly smaller than their CL limbs $(p=0.029$ and $p=0.026$, respectively) (Table 1; Figs. 5(a) and 5(c)). Following free mobilization, values for Injury I and Injury II were significantly smaller than their CL limbs for total ROM ( $p=0.0135$ and $p=0.0029$, respectively) and neutral zone length ( $p=0.004$ and $p=0.011$, respectively) (Table 1; Figs. $5(b)$ and $5(d))$. Pronation stiffness was only significantly increased for Injury II FM compared to CL $(p=0.034)$ (Table 2). Supination stiffness was significantly increased for Sham and Injury II IM compared to CL ( $p=0.025$ and $p=0.002$, respectively) (Table 2). There were no significant differences for any group in neutral zone stiffness (Table 2).

Correlations between P-S and flexion-extension for total ROM and neutral zone length values exhibited statistically significant and moderate correlations for IM animals $(p=0.0002, r=0.60$; $p<0.0001, r=0.63$, respectively) (Figs. 6(a) and 6(c)). For FM, the correlation between joint motions was statistically significant but weakly correlated for total ROM and neutral zone length ( $p=0.030, r=0.32 ; p=0.048, r=0.29$, respectively) (Figs. $6(b)$ and $6(d))$.

\section{Discussion}

This study shows that our hypothesis was partially confirmed because our rat elbow model of post-traumatic joint contracture exhibited impaired side-to-side function in $\mathrm{P}-\mathrm{S}$ motion that persisted long-term after joint free mobilization. P-S demonstrated a decreased total ROM and neutral zone length for injured limbs compared to uninjured CL limbs at both IM and FM. There was also little to no improvement in P-S when comparing IM to FM limbs; thus, after free joint mobilization, injured limbs never regained all lost motion when compared to CL. Contrary to our hypothesis, injured limbs did not exhibit significant differences compared to control limbs, and thus, $\mathrm{P}-\mathrm{S}$ is not as severely impacted in our animal model as flexion-extension. However, sideto-side differences (i.e., between limbs from the same animal) represent clinically relevant comparisons because a patient's range of motion in their injured forearm is typically compared to the motion of their uninjured arm rather than to that of a "normal" or control individual. Previously, animal models of knee contracture have compared the contralateral to the injured limb and thus demonstrate that the contralateral limb is an established control [18-20]. Therefore, the injury-immobilization protocol developed in our previous study not only induced joint mechanical changes that remained 
Immobilization

(a)

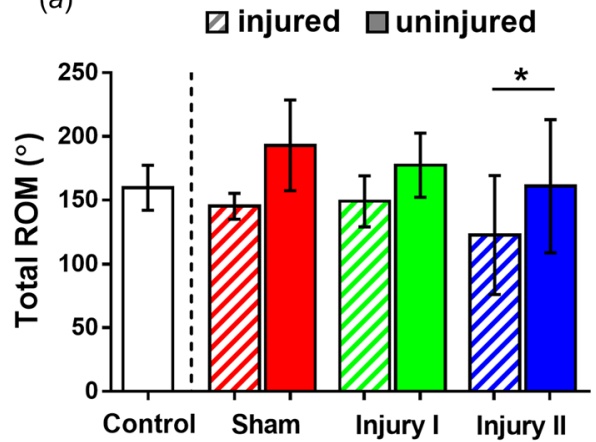

(c)

\section{$\square$ injured $\square$ uninjured}

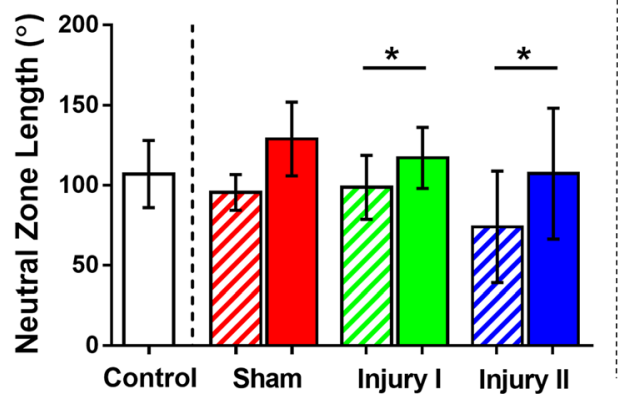

Free Mobilization

(b)

$\square$ injured $\square$ uninjured

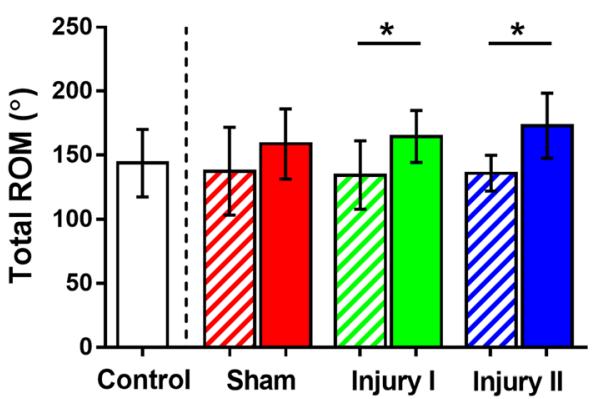

(d)

\section{$\square$ injured $\square$ uninjured}

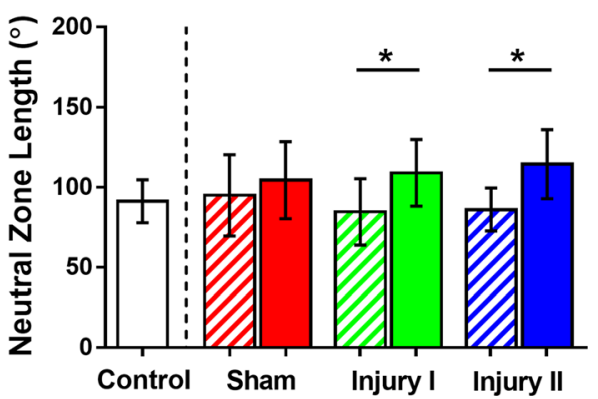

Fig. 5 Total range of motion (ROM) for (a) IM and (b) FM was decreased in injured limbs compared to contralateral (CL). Neutral zone length for (c) IM and (d) FM was also decreased compared to CL (average \pm standard deviation; diagonally shaded bars = injured; solid bars = contralateral; \#= significantly different from control; ${ }^{*}=$ significantly different from contralateral limb; $p<0.05)$.

Table 2 Quantitative results for pronation-supination joint mechanics: neutral zone, pronation and supination stiffness values for immobilization (IM) and free mobilization (FM)

\begin{tabular}{|c|c|c|c|c|c|c|}
\hline & \multicolumn{2}{|c|}{ Pronation stiffness (Nmm/deg) } & \multicolumn{2}{|c|}{ Supination stiffness (Nmm/deg) } & \multicolumn{2}{|c|}{ Neutral zone stiffness (Nmm/deg) } \\
\hline & Immobilization & Free mobilization & Immobilization & Free mobilization & Immobilization & Free mobilization \\
\hline Control & $0.58 \pm 0.11$ & $0.54 \pm 0.22$ & $0.45 \pm 0.12$ & $0.33 \pm 0.14$ & $0.019 \pm 0.008$ & $0.017 \pm 0.012$ \\
\hline Sham & $0.66 \pm 0.15$ & $0.48 \pm 0.21$ & $0.31 \pm 0.067$ & $0.54 \pm 0.12$ & $0.012 \pm 0.008$ & $0.017 \pm 0.018$ \\
\hline Sham CL & $0.41 \pm 0.16$ & $0.48 \pm 0.14$ & $0.43 \pm 0.12$ & $0.52 \pm 0.17$ & $0.011 \pm 0.007$ & $0.015 \pm 0.005$ \\
\hline Injury I & $0.69 \pm 0.27$ & $0.54 \pm 0.16$ & $0.35 \pm 0.12$ & $0.48 \pm 0.13$ & $0.013 \pm 0.004$ & $0.012 \pm 0.009$ \\
\hline Injury I CL & $0.44 \pm 0.28$ & $0.47 \pm 0.19$ & $0.54 \pm 0.21$ & $0.45 \pm 0.20$ & $0.008 \pm 0.010$ & $0.009 \pm 0.013$ \\
\hline Injury II & $0.78 \pm 0.37$ & $0.66 \pm 0.15$ & $0.29 \pm 0.087$ & $0.53 \pm 0.27$ & $0.024 \pm 0.018$ & $0.015 \pm 0.012$ \\
\hline Injury II CL & $0.47 \pm 0.50$ & $0.50 \pm 0.11$ & $0.57 \pm 0.080$ & $0.50 \pm 0.13$ & $0.012 \pm 0.012$ & $0.011 \pm 0.007$ \\
\hline
\end{tabular}

Note: Average \pm standard deviation; bolded values $=$ significantly different from contralateral limb; $p<0.05$.

No values were significantly different from control.

long-term in flexion-extension but also in P-S, mimicking longterm symptoms common to human patients [5-9,11].

P-S mechanical testing after IM found decreased total ROM, where Sham and Injury I lost $\sim 8 \mathrm{deg}$ and Injury II lost $\sim 23 \mathrm{deg}$ when compared to control (Fig. 5(a)). After free mobilization, the limbs did regain some motion compared to control; however, Injury I and Injury II remained decreased (Fig. 5(b)). Interestingly, when compared to $\mathrm{CL}$ the differences in total ROM lost for IM were Sham $\sim 25 \mathrm{deg}$, Injury I $\sim 16 \mathrm{deg}$, and Injury II $\sim 24 \mathrm{deg}$ (Fig. 5(a)). After free mobilization, total ROM loss persisted for Sham $\sim 13 \mathrm{deg}$, Injury I $\sim 18 \mathrm{deg}$, and Injury II $\sim 21 \mathrm{deg}$ when compared to CL (Fig. 5(b)). This data showed that side-to-side differences remain significantly decreased for total ROM after free mobilization for Injury I and Injury II. A similar trend was found for neutral zone length in $\mathrm{P}-\mathrm{S}$ when compared to either control or
CL (Figs. 5(c) and 5(d)). Clinically, a decreased neutral zone length shows that a limited amount of motion is possible before requiring a larger force to move the limb further, which means that the functional range of motion of the joint is decreased in the injured limbs. While there were significant changes in range of motion measurements, which are indicative of contracture, there were limited changes in all three stiffness measurements demonstrating that no persistent changes occurred in joint stiffness (Table 2). Different from our previous biomechanical results in flexion-extension $[5,6]$, for $\mathrm{P}-\mathrm{S}$ there is limited improvement after free mobilization compared to $\mathrm{CL}$, demonstrating that contracture is maintained longterm in this motion.

While there were only minor differences between the injury groups, there was a trend for increasing motion loss with increasing injury severity following free mobilization in our animal model of 


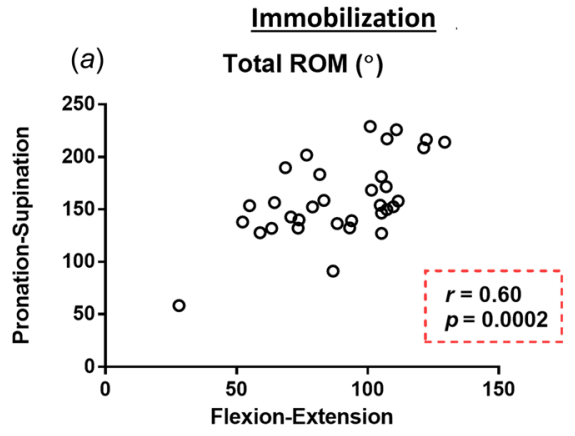

(c)

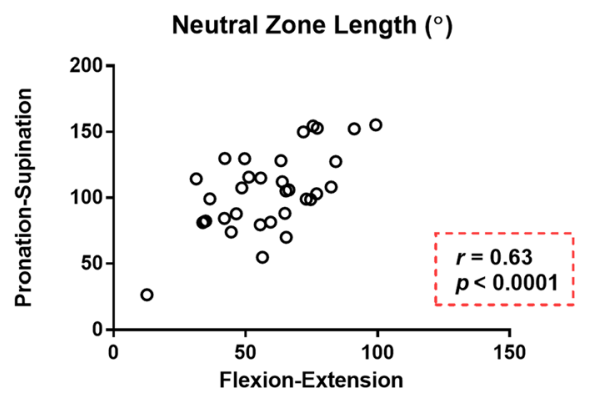

(b)

Free Mobilization

Total ROM $\left(^{\circ}\right)$

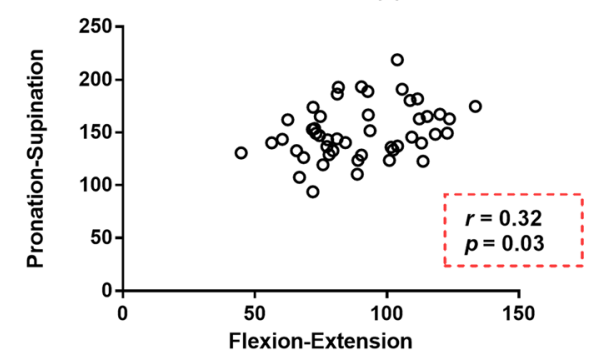

(d)

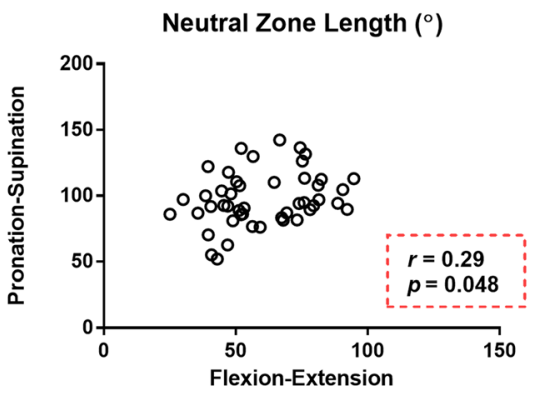

Fig. 6 Correlation between flexion-extension and pronation-supination for total range of motion (ROM) in (a) IM and (b) FM, and neutral zone length in (c) IM and (d) FM ( $p<0.05)$

elbow post-traumatic joint contracture. Similar to our previous results in flexion-extension [5,6], Injury II exhibited the most severe difference compared to its contralateral. Hence, these injurydependent differences have motivated our selection of utilizing the Injury II surgical protocol in future studies using this animal model.

An unexpected result in $\mathrm{P}-\mathrm{S}$ is that total ROM and neutral zone length of CL limbs were increased compared to control limbs, which were expected to be similar [19]. However, none of the CL limbs from injury groups were statistically different compared to control limbs (Fig. 5). Slight increases in mechanical parameters for $C L$ joints could be attributed to the animals compensating for the lost motion of the injured limb, especially since there is no body motion to compensate for P-S [21]. Therefore, the significant differences in total ROM and neutral zone length for IM and FM in this animal model were the result of an increase in CL limbs and a decrease in injured limbs rather than strictly being due to decreased parameter values in injured limbs (Fig. 5). It is also possible that the significant side-to-side differences after free mobilization are due to the injured limbs regaining motion similar to the controls, while the uninjured CL limbs maintained the motion gained during immobilization. Regardless of motion recovery, differences in P-S still exist in this animal model following free mobilization demonstrating that it has persistently altered side-to-side motion. A successful treatment for P-S motion would equilibrate the side-to-side differences, such that both limbs from the same animal exhibit similar motion.

We previously evaluated biological changes to the anterior joint capsule via histological analysis [5,6]. For IM and FM, the capsule exhibited increased adhesions and thickness compared to control and CL limbs. Since the anterior capsule extends proximally from the ulna coronoid process and radial fossa [21], increased adhesions and capsular thickness following injury could potentially inhibit the ability of the radial head to rotate during P-S, limiting total ROM. While we have not yet evaluated biological changes to the lateral collateral ligament following injury, scar tissue formed during healing could also potentially contribute to limited $\mathrm{P}-\mathrm{S}$.

While earlier studies of joint contracture using animal models have yielded significant insight, these models are limited anatomically and functionally for considering issues specific to the complex elbow joint. As many previous studies have used rat or rabbit knee models $[10,12,13,19]$, one limitation in these previous approaches was the ability to evaluate only flexion-extension motion. A majority of current surgical interventions (i.e., elbow arthroscopy, elbow contracture release, etc.) also primarily address flexion-extension motion; however, the forearm is capable of P-S, which cannot be fully compensated for by other arm or body movements [21]. Surgical intervention intending to improve P-S motion often requires invasive, open surgical procedures, which pose significant risk to nearby neurovascular structures [11]. The ability to evaluate both flexion-extension and $\mathrm{P}-\mathrm{S}$ in our model increases the clinical relevance compared to previous models and expands the range of potential studies that can be performed utilizing this model system. Research in a clinically relevant model of the elbow is needed to better understand the pathogenesis of contracture in a joint as complex as the elbow, so better prevention and treatment strategies can be developed for both types of motion.

Correlations between flexion-extension and P-S in total ROM and neutral zone length for both IM and FM exhibited a positive, significantly correlated relationship. Total ROM and neutral zone length IM exhibited moderate $r$ values demonstrating that these two types of motion display an overall increasing trend, where greater/ less motion in flexion-extension corresponds to greater/less motion in P-S. Both total ROM and neutral zone length FM had low $r$ values representing a weak relationship between flexion-extension and P-S after FM. Thus, FM does not affect flexion-extension and $\mathrm{P}-\mathrm{S}$ motion in the same way or to the same extent. In other words, improved mobility in one type of motion from FM does not guarantee similar improvement in other motion. Similarly, Ling et al. found that total ROM in flexion-extension and P-S was independent of each other after surgical release in human elbows and concluded that these motions should be managed as two separate entities [22]. Thus, it is imperative to study both types of motion and to consider each motion individually when developing treatment and rehabilitation strategies in the future.

This study is not without limitations. First, all mechanical testing was completed ex vivo and postmortem, which only represents the passive mechanical properties of tissues. Our group is currently investigating the active mechanical properties of muscle to evaluate any physiological and mechanical changes. During mechanical 
testing, it was difficult to perfectly align limbs in a neutral $(0 \mathrm{deg})$ starting position, so there is likely very small variation in the starting position. However, this would not affect total ROM, neutral zone length, and any stiffness values reported. Second, only one time point for free mobilization (i.e., 6 weeks) was evaluated. A previous study demonstrated that rat knees immobilized for 6.3 weeks and subsequently remobilized did not exhibit any additional improvement in range of motion after 5 weeks of free mobilization [23]; however, future studies could evaluate other time points of free mobilization to characterize the persistence of post-traumatic joint contracture symptoms in the elbow. Third, this study only evaluated male rats; however, we plan to evaluate female rats in the future.

\section{Conclusion}

In conclusion, this study demonstrates that our animal model of post-traumatic elbow contracture exhibits that motion is altered in $\mathrm{P}-\mathrm{S}$, although to a lesser extent than previously reported impairment to flexion-extension motion. To the best of our knowledge, this is the first time an animal model has been used to evaluate P-S motion. Side-to-side comparisons showed significantly decreased total ROM and neutral zone length of the injured limbs compared to uninjured, CL limbs. Weak correlations between flexion-extension and $\mathrm{P}-\mathrm{S}$ for free mobilization demonstrated that these different motions do not respond similarly to passive motion and further support the notion that both types of motion should be evaluated separately when developing a therapy/treatment strategy [11]. Future investigations will use this animal model to study which soft tissues surrounding the elbow contribute to contracture by evaluating active mechanics and genetic expression of muscle as well as capsular contribution to passive mechanics to understand which tissues should be targeted when developing a therapy for elbow contracture.

\section{Acknowledgment}

The authors thank the American Shoulder and Elbow Surgeons (ASES) for research funding and the National Institute of Health (NIH) for a training fellowship (NIBIB T32 EB018266) and research funding (NIAMS R03 ARR067504).

\section{Nomenclature}

$\mathrm{CL}=$ contralateral

$\mathrm{FM}=$ free mobilization

$\mathrm{IM}=$ immobilization

$\mathrm{P}-\mathrm{S}=$ pronation-supination

$\mathrm{ROM}=$ range of motion

\section{References}

[1] King, G. J., Morrey, B. F., and An, K.-N., 1993, "Stabilizers of the Elbow," J. Shoulder Elbow Surg., 2(3), pp. 165-174.

[2] Safran, M. R., and Baillargeon, D., 2005, "Soft-Tissue Stabilizers of the Elbow," J. Shoulder Elbow Surg., 14(1), pp. S179-S185.
[3] Cohen, M. S., Schimmel, D. R., Masuda, K., Hastings, H., and Muehleman, C. 2007, "Structural and Biochemical Evaluation of the Elbow Capsule After Trauma," J. Shoulder Elbow Surg., 16(4), pp. 484-490.

[4] Jawa, A., Jupiter, J. B., and Ring, D., 2012, "Pathogenesis and Classification of Elbow Stiffness," Operative Elbow Surgery, Churchill Livingstone/Elsevier, Edinburgh, UK, pp. 409-416.

[5] Lake, S. P., Castile, R. M., Borinsky, S., Dunham, C. L., Havlioglu, N., and Galatz, L. M., 2016, "Development and Use of an Animal Model to Study PostTraumatic Stiffness and Contracture of the Elbow," J. Orthop. Res., 34(2), pp. 354-364.

[6] Dunham, C. L., Castile, R. M., Havlioglu, N., Chamberlain, A. M., Galatz, L. M., and Lake, S. P., 2017, "Persistent Motion Loss After Free Joint Mobilization in a Rat Model of Post-Traumatic Elbow Contracture," J. Shoulder Elbow Surg., 26(4), pp. 611-618.

[7] Charalambous, C. P., and Morrey, B. F., 2012, "Posttraumatic Elbow Stiffness," J. Bone Jt. Surg., 94(15), pp. 1428-1437.

[8] Lindenhovius, A. L. C., and Jupiter, J. B., 2007, "The Posttraumatic Stiff Elbow: A Review of the Literature," J. Hand Surg., 32(10), pp. 1605-1623.

[9] Myden, C., and Hildebrand, K., 2011, "Elbow Joint Contracture After Traumatic Injury," J. Shoulder Elbow Surg., 20(1), pp. 39-44.

[10] Hildebrand, K. A., Holmberg, M., and Shrive, N., 2003, "A New Method to Measure Post-Traumatic Joint Contractures in the Rabbit Knee," ASME J. Biomech. Eng., 125(6), pp. 887-892.

[11] Keener, J. D., and Galatz, L. M., 2011, "Arthroscopic Management of the Stiff Elbow,” J. Am. Acad. Orthop. Surg., 19(5), pp. 265-274.

[12] Nesterenko, S., Morrey, M. E., Abdel, M. P., An, K.-N., Steinmann, S. P., Morrey, B. F., and Sanchez-Sotelo, J., 2009, "New Rabbit Knee Model of Posttraumatic Joint Contracture: Indirect Capsular Damage Induces a Severe Contracture," J. Orthop. Res., 27(8), pp. 1028-1032.

[13] Trudel, G., and Uhthoff, H. K., 2000, "Contractures Secondary to Immobility: Is the Restriction Articular or Muscular? An Experimental Longitudinal Study in the Rat Knee," Arch. Phys. Med. Rehabil., 81(1), pp. 6-13.

[14] Whishaw, I. Q., and Pellis, S. M., 1990, "The Structure of Skilled Forelimb Reaching in the Rat: A Proximally Driven Movement With a Single Distal Rotatory Component," Behav. Brain Res., 41(1), pp. 49-59.

[15] Whishaw, I. Q., Gorny, B., Foroud, A., and Kleim, J. A., 2003, "Long-Evans and Sprague-Dawley Rats Have Similar Skilled Reaching Success and Limb Representations in Motor Cortex but Different Movements: Some Cautionary Insights Into the Selection of Rat Strains for Neurobiological Motor Research," Behav. Brain Res., 145(1), pp. 221-232.

[16] Sacrey, L.-A. R., Alaverdashvili, M., and Whishaw, I. Q., 2009, "Similar Hand Shaping in Reaching-for-Food (Skilled Reaching) in Rats and Humans Provides Evidence of Homology in Release, Collection, and Manipulation Movements," Behav. Brain Res., 204(1), pp. 153-161.

[17] Aoki, S., Sato, Y., and Yanagihara, D., 2012, "Characteristics of Leading Forelimb Movements for Obstacle Avoidance During Locomotion in Rats," Neurosci. Res., 74(2), pp. 129-137.

[18] Trudel, G., Uhthoff, H. K., Goudreau, L., and Laneuville, O., 2014 "Quantitative Analysis of the Reversibility of Knee Flexion Contractures With Time: An Experimental Study Using the Rat Model," BMC Musculoskeletal Disord., 15(1), p. 338.

[19] Abdel, M. P., Morrey, M. E., Grill, D. E., Kolbert, C. P., An, K.-N., Steinmann, S. P., Sanchez-Sotelo, J., and Morrey, B. F., 2012, "Effects of Joint Contracture on the Contralateral Unoperated Limb in a Rabbit Knee Contracture Model: A Biomechanical and Genetic Study," J. Orthop. Res., 30(10), pp. 1581-1585.

[20] Abdel, M. P., Morrey, M. E., Barlow, J. D., Kreofsky, C. R., An, K.-N., Steinmann, S. P., Morrey, B. F., and Sanchez-Sotelo, J., 2012, "Myofibroblast Cells Are Preferentially Expressed Early in a Rabbit Model of Joint Contracture," J. Orthop. Res., 30(5), pp. 713-719.

[21] Bryce, C. D., and Armstrong, A. D., 2008, "Anatomy and Biomechanics of the Elbow," Orthop. Clin. North Am., 39(2), pp. 141-154.

[22] Ling, S. K. K., Lui, T. H., Faan, Y. S., Lui, P. W. Y., and Ngai, W. K., 2014, "Post-Traumatic Elbow Rotational Stiffness," Shoulder Elbow, 6(2), pp. $119-123$.

[23] Evans, E. B., Eggers, G. W. N., Butler, J. K., and Blumel, J., 1960, "Experimental Immobilization and Remobilization of Rat Knee Joints," J. Bone Jt. Surg. Am., 42(5), pp. 737-758. 